\title{
DNVF-Memorandum III - Methoden für die Versorgungsforschung, Teil 4 - Konzept und Methoden der organisationsbezogenen Versorgungsforschung: Kurzfassung
}

\author{
DNVF-Memorandum III - Methods for Health Services Research, \\ Part 4 - Concept and Methods for Organizational Health Services \\ Research: Short Version
}

\section{(ㄷ)(1) (8) $\odot$}

\author{
Autoren \\ Lena Ansmann 1, 2, Ute-Susann Albert' ${ }^{3}$, Ramona Auer ${ }^{4}$, Walter Baumann5, Eva Maria Bitzer6, Martina Bögel7, \\ Nicole Ernstmann ${ }^{8}$, Johannes Gostomzyk ${ }^{9}$, Katja Götz ${ }^{10}$, Ursula Hahn ${ }^{11},{ }^{12}$, Antje Hammer ${ }^{13}$, Alfons Hollederer ${ }^{14}$, \\ Kira Isabel Hower ${ }^{2}$, Tanja Kostuj ${ }^{15}$, Christoph Kowalski ${ }^{16}$, Stefan Nöst ${ }^{17,}{ }^{18}$, Marina Nowak², Holger Pfaff ${ }^{2}$, \\ Lars Rölker-Denker ${ }^{1}$, Horst Christian Vollmar ${ }^{19}$, Markus Antonius Wirtz ${ }^{20}$
}

Institute

1 Department für Versorgungsforschung, Fakultät für Medizin und Gesundheitswissenschaften, Carl von Ossietzky Universität Oldenburg, Oldenburg

2 Humanwissenschaftliche Fakultät und Medizinische Fakultät der Universität zu Köln, Institut für Medizinsoziologie, Versorgungsforschung und Rehabilitationswissenschaften, Köln

3 Medizinische Fakultät, Philipps-Universität Marburg, c/o AWMF-Arbeitsgemeinschaft der Wissenschaftlichen Medizinischen Fachgesellschaften e.V., Marburg

4 AOK Baden-Württemberg, Fachbereich Versorgungsmanagement, Stuttgart

5 Wissenschaftliches Institut der Niedergelassenen Hämatologen und Onkologen, (WINHO GmbH), Köln

6 Institut für Alltagskultur, Bewegung und Gesundheit, Padagogische Hochschule Freiburg, Freiburg im Breisgau

7 Deutsche Gesellschaft für Schlafforschung und Schlafmedizin (DGSM), Geschäftsstelle, Schwalmstadt-Treysa

8 Klinik und Poliklinik für Psychosomatische Medizin und Psychotherapie, Forschungsstelle für Gesundheitskommunikation und Versorgungsforschung, Universitätsklinikum Bonn, Bonn

9 Deutsche Gesellschaft für Sozialmedizin und Prävention e. V., Mannheim

10 Institut für Allgemeinmedizin, Universitätsklinikum Schleswig Holstein, Campus Lübeck, Lübeck

11 Fakultät für Gesundheit der Universität Witten/ Herdecke, Institut für Medizinische Biometrie und Epidemiologie, Witten

12 OcuNet GmbH \& Co KG, Geschäftsführung, Düsseldorf

13 Institut für Patientensicherheit, Universitätsklinik Bonn, Bonn
14 Bayerisches Landesamt fur Gesundheit und Lebensmittelsicherheit, Sachgebiet GE6 Versorgungsqualität, Gesundheitsökonomie, Gesundheitssystemanalyse, Nürnberg

15 Klinik für Orthopädie und Unfallchirurgie, Klinikum Lippe, Lemgo

16 Deutsche Krebsgesellschaft e.V., Zertifizierung, Berlin

17 Duale Hochschule Baden-Württemberg, Studienfachbereich Gesundheit, Angewandte Gesundheits- und Pflegewissenschaften, Stuttgart

18 Abteilung Allgemeinmedizin und Versorgungsforschung, Universitätsklinikum Heidelberg

19 Medizinische Fakultät, Abteilung für Allgemeinmedizin, Ruhr-Universität Bochum, Bochum

20 Institut für Psychologie, Pädagogische Hochschule Freiburg, Freiburg

Schlüsselwörter

Organisationsbezogene Versorgungsforschung, Memorandum, Versorgungsorganisation, Methoden, Evaluation, Implementierung

Key words

Organizational health services research, Memorandum, healthcare organizations, methods, evaluation, implementation

Bibliografie

DOI https://doi.org/10.1055/a-0862-0407

Gesundheitswesen 2019; 81: 220-224

(c) Georg Thieme Verlag KG Stuttgart · New York

ISSN 0941-3790 


\section{Korrespondenzadresse}

Prof. Lena Ansmann

Department für Versorgungsforschung

Abteilung Organisationsbezogene Versorgungsforschung

Carl von Ossietzky Universität Oldenburg

Ammerländer Heerstraße 140

26129 Oldenburg

lena.ansmann@uni-oldenburg.de

Hinweis für unsere Leser: Die Langfassung der drei Kapitel des Memorandums finden Sie im Online-Archiv von „Das Gesundheitswesen“ zum freien Download. https://doi.org/10.1055/a-0862-0527

https://doi.org/10.1055/a-0862-0565

https://doi.org/10.1055/a-0862-0588

\section{ZUSAMMENFASSUNG}

Das Forschungsfeld der organisationsbezogenen Versorgungforschung ist von zunehmender Bedeutung. Ausgehend von der Vertiefung zu Methoden der organisationsbezogenen Versorgungsforschung des Memorandums III, Teil 1 aus dem Jahr 2009 werden die weiterentwickelten Standards und Grundlagen zusammengefasst. Das Memorandum behandelt die theoretische Rahmung, grundlegende Methodenansätze und Methoden zur Konzeption, Evaluation und Implementierung komplexer Interventionen in Versorgungsorganisationen.

\section{ABSTRACT}

Organizational health services research in Germany is of increasing relevance. Based on the guide on methods for organizational health services research of the Memorandum III, part 1 from the year 2009, the fundamentals and standards have now been refined. The memorandum captures the theoretical framework, basic methodological approaches and methods in health services research for the design, evaluation and implementation of complex interventions in healthcare organizations.

\section{Einleitung}

Das Forschungsfeld der organisationsbezogenen Versorgungforschung ist in Deutschland noch relativ jung und von zunehmender Bedeutung. Das Deutsche Netzwerk Versorgungsforschung e. V. (DNVF) hat im Jahr 2009 - getragen von den im DNVF organisierten Fachgesellschaften und Mitgliedern - eine Vertiefung zu „Methoden der organisationsbezogenen Versorgungsforschung“ des Memorandums III, Teil 1 konsentiert [1]. Ausgehend von dieser Veröffentlichung und dem Bedeutungsgewinn des Forschungsfeldes ist eine Weiterentwicklung der konzeptionellen und methodischen Grundlagen und Standards erforderlich. Die Neuauflage und Erweiterung der Veröffentlichung aus dem Jahr 2009 widmet sich in 3 Kapiteln den Fragen: (1) Was sind die konzeptionellen und theoretischen Grundlagen der organisationsbezogenen Versorgungsforschung und wie lassen sich diese definieren? (2) Welche Zielgrößen, Datenquellen, Erhebungs- und Analysemethoden sind für die organisationsbezogene Versorgungsforschung besonders wichtig und geeignet? (3) Welche methodischen Ansätze eignen sich für Studien zur Konzeption, Evaluation und Implementierung komplexer Interventionen in Versorgungsorganisationen? Die Veröffentlichung aus dem Jahr 2009 wurde dabei in der vorliegenden Neufassung um wichtige konzeptuelle und methodische Inhalte erweitert. Diese umfassen die organisationstheoretische und konzeptuelle Rahmung sowie erstmals eine Definition der organisationsbezogenen Versorgungsforschung. Der methodische Ansatz wurde geschärft, indem die Notwendigkeit von Mixed Methods hervorgehoben und die Möglichkeiten der Nutzung bzw. Erhebung von Primär- und Sekundärdaten in diesem Bereich aufgezeigt werden. Es wird ein weiterentwickeltes Phasenmodell zur Evaluation komplexer Interventionen unter Berücksichtigung des organisationalen Kontextes präsentiert, das Methoden des Interventionsdesign, der Wirksamkeits- und Wirkungsforschung sowie der Implementierungsforschung integ- riert. Im Folgenden werden in einer Kurzfassung die Inhalte der $3 \mathrm{Ka}$ pitel und die zentralen Definitionen und Aussagen vorgestellt. Für das tiefere Verständnis und für Literaturhinweise ist es jedoch notwendig, die Online-Langversionen der 3 Kapitel zu lesen. Im Anhang wird zudem das Vorgehen bei der Entwicklung, Kommentierung und Abstimmung des Memorandums beschrieben und die Mitglieder und Mitgliedsorganisationen des DNVF genannt, von denen das Memorandum getragen wird.

\section{Ergebnisse}

\section{Kapitel 1 - Definition und Konzept der organisationsbezogenen Versorgungsforschung}

Im ersten Kapitel [2] erfolgt eine theoretische Rahmung und die Begrifflichkeiten und Aufgaben der organisationsbezogenen Versorgungsforschung werden definiert. Das Kapitel gibt zunächst eine Übersicht über Organisationstheorien und arbeitet dann die Besonderheiten von Versorgungsorganisationen heraus. Danach werden Aufgaben der organisationsbezogenen Versorgungsforschung definiert sowie Wechselwirkungen der Organisationsebene mit der Individual- und Systemebene konzeptualisiert. Es wurde die folgende Definition der organisationsbezogenen Versorgungsforschung entwickelt:

Die organisationsbezogene Versorgungsforschung befasst sich mit

1) denRahmenbedingungen, unterdenen Versorgungsorganisationen agieren und deren Wechselwirkungen auf Individuumsund Organisationsebene,

2) den Strukturen, Prozessen und Kulturen von Versorgungsorganisationen, 
3) den Interaktionen innerhalb und zwischen Versorgungsorganisationen und

4) den Auswirkungen dieser Faktoren auf das Ergebnis und die Organisation gesundheitlicher Versorgung.

In verschiedenen Disziplinen sind vielfältige Definitionen, Modelle und Theorien über das Funktionieren von Organisationen entstanden. Diese Vielfalt kann genutzt werden, um Forschungsfragen, Methoden und Analyseverfahren für die organisationsbezogene Versorgungsforschung abzuleiten. Hierbei sollten Fragestellungen und Methoden angewendet werden, die die Komplexität des Forschungsgegenstandes angemessen berücksichtigen. Dies sollte zum einen durch ein besonderes Augenmerk auf die Wechselwirkungen der Mesoebene mit der Mikro- und Makroebene realisiert werden. Zum anderen sollte es neben der Identifikation von Unterschieden in der Versorgungsqualität zwischen Versorgungsorganisationen auch Ziel der organisationsbezogenen Versorgungsforschung sein, zu verstehen, wodurch diese Unterschiede zu erklären sind und über welche Mechanismen organisationale Interventionen zu Veränderungen führen.

\section{Kapitel 2 - Methodische Ansätze der organisationsbezogenen Versorgungsforschung: Zielgrößen, Datenquellen, Datenerhebung und Datenanalyse}

Ziel des zweiten Kapitels [3] ist es, die methodischen Besonderheiten mit ihren Konsequenzen für die Datenerhebungen, -nutzung und -analyse zu umreißen. In dem Kapitel werden zum einen verschiedene Arten von für die organisationsbezogene Versorgungsforschung relevanten Zielgrößen beschrieben. Zum anderen wird der methodische Ansatz des Forschungszweiges herausgearbeitet, der die Relevanz von Mixed Methods betont, um Versorgungsunterschiede zwischen Organisationen erklären zu können. Zudem werden besonders geeignete Datenquellen, Erhebungs- und Auswertungsmethoden skizziert. In der Versorgungsforschung ist die Sicht auf organisationale Zusammenhänge bisher vielfach noch versperrt durch die Unkenntnis geeigneter theoretischer und methodischer Ansätze. So wird der Organisationsbezug von Forschungsfragen und -daten häufig nicht erkannt. Die Potenziale der organisationsbezogenen Versorgungsforschung bleiben dadurch oft ungenutzt. Durch die Ergänzung der outcomebezogenen Zielgrößen um Größen aus dem Bereich der organisationalen Versorgungsstrukturen, -prozesse und -kulturen ist die organisationsbezogene Versorgungsforschung in der Lage, die vielfach beschriebene „Black Box“ zu beleuchten. Denn sie kann potenziell die Variation von Patientenoutcomes ${ }^{1}$ zwischen Versorgungsorganisationen und die Wirkungsweise von Interventionen in der Versorgung erklären. Um dies leisten zu können, ist die Nutzung geeigneter Datenquellen sowie die Anwendung und Integration geeigneter Methoden der Datenerhebung, -nutzung und -analyse erforderlich.

\section{Kapitel 3 - Methodische Ansätze zur Evaluation und Implementierung komplexer Interventionen in Versorgungsorganisationen}

Das dritte Kapitel [4] behandelt Kriterien und Standards für Methoden der Konzeption, Evaluation und Implementierung komplexer Interventionen in Versorgungsorganisationen. In der Versorgungsforschung sind alle interventionellen, empirischen Vorhaben unter der Perspektive der Qualität des Interventionsdesigns, der Analyse von Wirkprozessen, des Wirksamkeitsnachweises und der bestmöglichen Implementierbarkeit zu betrachten. Hierbei wird den Besonderheiten des organisationalen Kontextes besondere Bedeutung zugeschrieben. Die theorie- und evidenzbasierte Wahl und Gestaltung der Intervention erfolgen in der Phase des Interventionsdesigns. Die Wirksamkeits- und Wirkungsforschung prüft die empirischen Effekte der Intervention auf patientenrelevante Outcomes gemäß forschungsmethodisch solider Kriterien. Die Implementierungsforschung beschäftigt sich damit, wie komplexe Interventionen an die lokalen Rahmenbedingungen angepasst werden können, sodass die Wirksamkeit der Intervention gewährleistet bleibt. Entsprechend muss die Evidenz für den Nutzen von Interventionen in der organisationsbezogenen Versorgungsforschung gemäß der Inhalte und Anforderungen aller 3 Bereiche geprüft und sichergestellt werden. Strukturen und koordinierte Prozessabläufe in und zwischen organisationalen Einheiten und Ebenen müssen in der organisationsbezogenen Versorgungsforschung besonders berücksichtigt werden. Eine valide Beschreibung des Handelns Einzelner und der patientenrelevanten Versorgungsprozesse bedarf stets einer analytischen Reflexion im sozialen und organisationalen (Funktions-) Kontext.

\section{Diskussion}

Das Memorandum bietet eine konzeptionelle und methodische Grundlage für die organisationsbezogene Versorgungsforschung, die gemeinsam mit Experten und unter Einbezug einschlägiger und aktueller internationaler Literatur erarbeitet worden ist. Die kritischen Rückmeldungen und Vorschläge im Rahmen des Konsentierungsprozesses des DNVF e.V. wurden bestmöglich und transparent eingearbeitet. Die vorgestellten methodologischen Grundlagen sind als Methodeninventar zu verstehen. Sie sollen zum einen zu einer stärkeren Berücksichtigung des organisationalen Kontextes in der Versorgungsforschung beitragen und zum anderen dazu genutzt werden, die konzeptionelle und methodische Qualität von Untersuchungen der organisationsbezogenen Versorgungsforschung zu sichern, weiterzuentwickeln und zu verbessern.

Interessenkonflikt

Die Autoren geben an, dass kein Interessenkonflikt besteht.

1 Obwohl aus Gründen der besseren Lesbarkeit im Text die männliche Form gewählt wurde, beziehen sich alle Angaben auf alle Geschlechter. 
[1] Pfaff H, Albert U-S, Bornemann R et al. Methoden für die organisationsbezogene Versorgungsforschung. Gesundheitswesen 2009; 71: 777-790

[2] Ansmann L, Baumann W, Gostomzyk J et al. DNVF-Memorandum III Methoden für die Versorgungsforschung, Teil 4 - Konzept und Methoden der organisationsbezogenen Versorgungsforschung, Kapitel 1 - Definition und Konzept der organisationsbezogenen Versorgungsforschung. Gesundheitswesen 2019; DOI 10.1055/a-0862-0527

[3] Rölker-Denker L, Kowalski C, Ansmann L et al. DNVF-Memorandum III - Methoden für die Versorgungsforschung, Teil 4 - Konzept und Methoden der organisationsbezogenen Versorgungsforschung, Kapitel 2 - Methodische Ansätze der organisationsbezogenen Versorgungsforschung: Zielgrößen, Datenquellen, Datenerhebung und Datenanalyse. Gesundheitswesen 2019; DOI 10.1055/a-0862-0565

[4] Wirtz MA, Bitzer EM, Albert US et al. DNVF-Memorandum III - Methoden für die Versorgungsforschung, Teil 4 - Konzept und Methoden der organisationsbezogenen Versorgungsforschung, Kapitel 3 - Methodische Ansätze zur Evaluation und Implementierung komplexer Interventionen in Versorgungsorganisationen. Gesundheitswesen 2019; DOI 10.1055/a-0862-0588

\section{Entwicklung, Kommentierung und Abstimmung des Memorandums}

Nach den Standards der Erstellung von Memoranden des DNVF wurde die Zustimmung des DNVF-Vorstands zur Erarbeitung eines Memorandums eingeholt und alle Mitglieder und Mitgliedsorganisationen wurden zur Mitarbeit eingeladen. Es haben sich 20 Personen aktiv an der Erstellung des Memorandums beteiligt (Autorengruppe). Die Autorengruppe stimmte die Grundstruktur des Memorandums ab, bildete für jedes der 3 Kapitel eine Autorengruppe und erstellte gemeinsam den Entwurf des Memorandums. Struktur und Inhalt wurden auf dem Deutschen Kongress für Versorgungsforschung in 2017 und 2018 diskutiert. Alle Mitglieder- und Mitgliedsorganisationen des DNVF hatten in einer 3-monatigen Kommentierungsphase vom 10.07. bis 10.10.2018 die Möglichkeit, zum Entwurf Stellung zu nehmen. Auf die Kommentierungen wurde in einer finalen Überarbeitung vollumfänglich reagiert. Danach wurde eine finale Fassung erstellt, die an die ordentlichen sowie an die korrespondierenden institutionellen Mitglieder des DNVF mit der Einladung zur Mitzeichnung versandt wurde. Die Autoren des aktuellen Memorandums möchten sich bei allen am Konsentierungsprozess Beteiligten für ihre konstruktiven Diskussionsbeiträge bedanken.

Folgende Fachgesellschaften haben sich im Rahmen des Kommentierungsprozesses am Memorandum zu Konzept und Methoden der organisationsbezogenen Versorgungsforschung beteiligt:

- Deutsche Gesellschaft für Gynäkologie und Geburtshilfe

- Deutsche Gesellschaft für Pflegewissenschaft

- Deutsche Gesellschaft für Prävention und Rehabilitation von Herz-Kreislauferkrankungen

- Deutsche Gesellschaft für Rehabilitationswissenschaft

- Deutsche Gesellschaft für Senologie

Folgende Wissenschaftliche Institute und Forschungsverbünde haben sich im Rahmen des Kommentierungsprozesses am Memorandum zu Konzept und Methoden der organisationsbezogenen Versorgungsforschung beteiligt:

- Centre for Health and Society

- LVR-Institut für Versorgungsforschung

Folgende persönliche Mitglieder des DNVF haben sich im Rahmen des Kommentierungsprozesses am Memorandum zu Konzept und Methoden der organisationsbezogenen Versorgungsforschung beteiligt:

- Eugenia Larjow

Das Memorandum zu Konzept und Methoden der organisationsbezogenen Versorgungsforschung wird von folgenden ordentlichen und korrespondierenden institutionellen Mitgliedern des Deutschen Netzwerks Versorgungsforschung e. V. getragen.

Von folgenden Mitgliedern der Sektion „Fachgesellschaften“:

- Deutsche Diabetes Gesellschaft e.V.

- Deutsche Gesellschaft für Allgemeinmedizin

- Deutsche Gesellschaft für Ambulante Allgemeine Pädiatrie

- Deutsche Gesellschaft für Anästhesiologie und Intensivmedizin e.V. 
- Deutsche Gesellschaft für Angiologie - Gesellschaft für Gefäßmedizin e.V.

- Deutsche Gesellschaft für Arbeitsmedizin und Umweltmedizin e.V.

- Deutsche Gesellschaft für Gynäkologie und Geburtshilfe e.V.

- Deutsche Gesellschaft für Hämatologie und Medizinische Onkologie e.V.

- Deutsche Gesellschaft für Innere Medizin e.V.

- Deutsche Gesellschaft für Kardiologie - Herz- und Kreislaufforschung e.V.

- Deutsche Gesellschaft für Kinder- und Jugendmedizin e.V.

- Deutsche Krebsgesellschaft e.V.

- Deutsche Gesellschaft für Medizinische Soziologie e.V.

- Deutsche Gesellschaft für Mund-, Kiefer und Gesichtschirurgie

- Deutsche Gesellschaft für Neurochirurgie e.V.

- Deutsche Gesellschaft für Neurologie e.V.

- Deutsche Gesellschaft für Orthopädie und Orthopädische Chirurgie e.V.

- Deutsche Gesellschaft für Psychiatrie und Psychotherapie, Psychosomatik und Nervenheilkunde e.V.

- Deutsche Gesellschaft für Rehabilitationswissenschaften e.V.

- Deutsche Gesellschaft für Schlafforschung und Schlafmedizin e.V.

- Deutsche Gesellschaft für Senologie e.V.

- Deutsche Gesellschaft für Sozialmedizin und Prävention e.V.

- Deutsche Gesellschaft für Zahn-, Mund- und Kieferheilkunde e.V.

- Deutsche Ophtalmologische Gesellschaft e.V.

- Deutsche Sepsis-Gesellschaft e.V.

Von folgenden Mitgliedern der Sektion „Wissenschaftliche Institute und Forschungsverbände“:

- Centre for Health and Society, Universitätsklinikum Düsseldorf

- Center for Health Care Research, Universitätsklinikum Hamburg-Eppendorf
- Gesundheit Österreich GmbH

- Institut für Hausarztmedizin der Universität Bonn

- Institut für Medizinsoziologie, Versorgungsforschung und Rehabilitationswissenschaft der Humanwissenschaftlichen Fakultät und der Medizinischen Fakultät der Universität zu Köln (KöR)

- Institut für Patientensicherheit, Universitätsklinikum Bonn

- Interdisziplinäres Zentrum für Versorgungsforschung im Gesundheitswesen der Universität Witten/Herdecke

- Landesinstitut Gesundheit des Bayerischen Landesamts für Gesundheit und Lebensmittelsicherheit

- LVR-Institut für Versorgungsforschung

- Medizinische Hochschule Brandenburg Theodor Fontane

- Sektion Versorgungsforschung und Rehabilitationsforschung, Universitätsklinikum Freiburg

- Wissenschaftliches Institut der Niedergelassenen Hämatologen und Onkologen $\mathrm{GmbH}$

- Zentrum für Evidenzbasierte Gesundheitsversorgung, Universitätsklinikum Carl Gustav Carus an der TU Dresden

- Zentrum für Versorgungsforschung Köln

- Universität Potsdam, Professur für Rehabilitationswissenschaften

Von folgenden Mitgliedern der Sektion „Juristische Personen und Personenvereinigungen“:

- Berufsverband der Kinder- und Jugendärzte e.V.

- Deutscher Verband für Gesundheitssport und Sporttherapie e.V.

- Kassenärztliche Bundesvereinigung

- OptiMedis AG

- 4QD - Qualitätskliniken.de GmbH 